\title{
Cathodoluminescence mapping for the determination of n-type doping in single GaAs nanowires
}

\author{
Hung-Ling Chen ${ }^{1}$, Chalermchai Himwas ${ }^{1}$, Andrea Scaccabarozzi ${ }^{1,2}$, Pierre Rale ${ }^{1}$, Fabrice Oehler ${ }^{1}$, Aristide \\ Lemaître $^{1}$, Laurent Lombez ${ }^{2,3}$, Jean-François Guillemoles ${ }^{2,3}$, Maria Tchernycheva ${ }^{1}$, Jean-Christophe \\ Harmand $^{1}$, Andrea Cattoni ${ }^{1}$, Stéphane Collin ${ }^{1,2}$ \\ ${ }^{1}$ Centre of Nanoscience et de Nanotechnology, CNRS, University Paris-Sud/Paris-Saclay, Marcoussis, \\ France \\ ${ }^{2}$ Institut Photovoltaïque d'Ile-de-France (IPVF), Antony, France \\ ${ }^{3}$ Institut de Recherche et Développement sur l'Energie Photovoltaïque (IRDEP) EDF/CNRS/Chimie Paris \\ Tech, Chatou, France
}

\begin{abstract}
We present a new method to determine the doping level of n-type semiconductors at the nanoscale. Lowtemperature and room-temperature cathodoluminescence (CL) measurements are carried out on single Si-doped GaAs nanowires. The spectral shift and the broadening of luminescence spectra are a signature of an increased density of electrons. They are compared to CL spectra of well-calibrated planar Si-doped GaAs layers whose doping levels are determined by Hall measurements and compared to previous experimental studies. We infer a n-type doping of $1 \times 10^{18} \mathrm{~cm}^{-3}$ to $2 \times 10^{18} \mathrm{~cm}^{-3}$, with a high spatial homogeneity along the nanowire. These results show that cathodoluminescence provides an alternative way to probe carrier concentration in nanostructured and polycrystalline semiconductors, and to map the spatial inhomogeneity of dopants.
\end{abstract}

\section{INTRODUCTION}

Semiconductor nanowires provide a new route toward low cost photovoltaics and multi-junction architectures. They present natural light-trapping properties. Their small lateral dimension allows lattice mismatch growth and enables direct integration of III-V nanowires on silicon cells, or to fabricate flexible devices [1,2]. However, controlling the doping is a major issue in the fabrication of nanowire-based solar cells. It is especially crucial for core-shell structures due to small radial dimension, and numerical calculations have shown that the requirement of doping level is higher for radial junction than axial junction solar cells $[3,4]$.

Hall measurement is the conventional method used for the characterization of doping in planar semiconductor layers. For nanowires, electrical methods can still be applied $[5,6]$. However, contacting a single nanowire and forming good ohmic contacts requires considerable technical efforts and can hardly be applied to a large number of nanowires. Contactless optical methods based on terahertz spectroscopy and photoluminescence have shown usefulness in measuring the doping level of ensembles of nanowires [7,8], but they cannot be used to assess the homogeneity of semiconductor nanostructures and for the characterization of single nanowires.
In this work, we show that cathodoluminescence (CL) can be used to assess the doping level of n-type GaAs at the nanoscale. CL maps of single GaAs nanowires are measured at $20 \mathrm{~K}$ and room temperature. They show a high homogeneity along the wire, and the peaks of CL spectra shifting to higher energy (Burstein-Moss shift) is a signature of increased electron concentration. We compare CL spectra of single nanowires with CL spectra of planar Si-doped GaAs with well-calibrated doping levels, and with data published in previous studies. We infer electron concentrations in GaAs nanowires of $1-2 \times 10^{18} \mathrm{~cm}^{-3}$.

\section{II. -EXPERIMENTAL}

GaAs nanowires were grown by molecular beam epitaxy (MBE) on $\mathrm{Si}(111)$ substrate. Un-intentionally doped GaAs nanowires were firstly grown by self-catalyzed vapor-liquidsolid method. Ga catalyst droplet was then crystallized by exposing the sample to As flux only. Finally, Si-doped GaAs shell was grown using standard conditions similar to planar films. Nanowire length was measured to be 3-4 $\mu \mathrm{m}$ and diameter about $200 \mathrm{~nm}$. Nanowires are dispersed on a $\mathrm{Si}$ substrate for CL measurements.

Cathodoluminescence (CL) measurements were performed with an Attolight "Chronos" quantitative cathodoluminescence microscope. The mean electron current is of the order of $1 \mathrm{nA}$ and the acceleration voltage is $6 \mathrm{kV}$. CL spectra were recorded on an Andor Newton CCD camera with a Horiba dispersive spectrometer (grating: 150 grooves $/ \mathrm{mm}$ ). Light is collected through an achromatic reflective objective with a numerical aperture of 0.72 . Luminescence spectra are corrected for the optical response of the collection and detection system.

\section{RESULTS}

\section{A. Cathodoluminescence mapping}




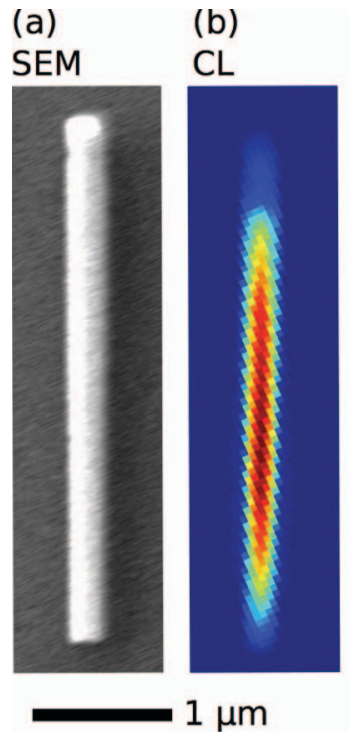

(c)

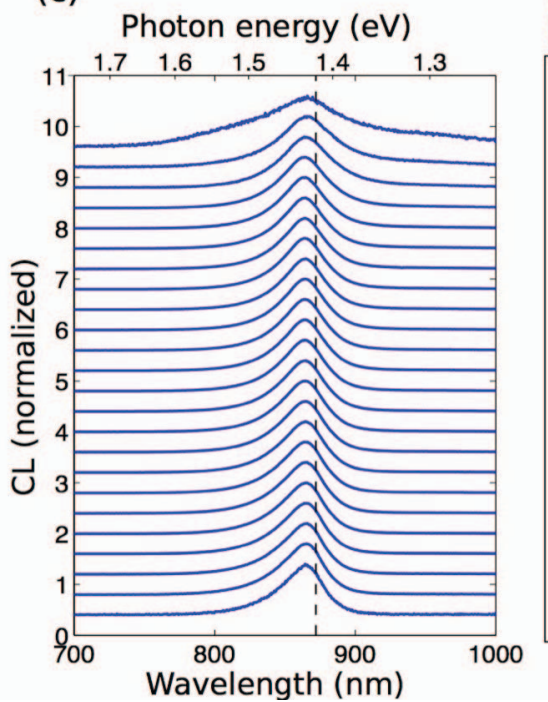

(d)

Peak energy

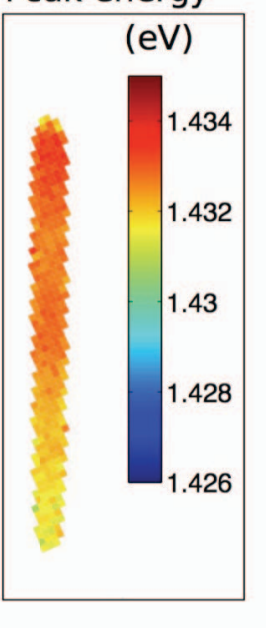

(e)

FWHM

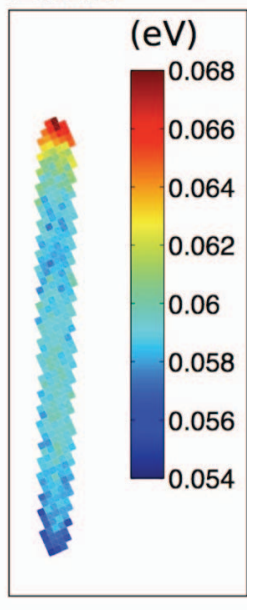

Fig. 1. Cathodoluminescence mapping of a single GaAs nanowire. (a) SEM image of the nanowire. (b) CL map of the total luminescence intensity integrated over the whole emission spectral range. (c) CL emission spectra along the nanowire showing a very homogeneous emission except close to the tip, where the Ga catalyst droplet was crystallized. Doping analysis is done in the homogeneous part of the nanowire. (d) Map of the peak energy of luminescence emission. (e) Map of the full width at half maximum (FWHM) of the CL peaks.

Figure 1 shows cathodoluminescence measurements of a single GaAs nanowire dispersed on a Si substrate. The CL intensity is constant along the major part of the nanowire, except at the nanowire tip (top of the images in Figure 1). This part has been grown by consuming the liquid Ga catalyst droplet, and changing the contact angle of liquid droplet typically produces different crystal phases (zinc-blende or wurtzite) [9]. A broadening of CL spectra is observed in this region (Figure 1c). In the following, we will only analyze CL spectra from the homogeneous region. Figures 1(d) and (e) show the maps of the luminescence peak energy and full width at half mawimum (FWHM).

\section{B. Low-temperature and room-temperature CL spectra}

Low-temperature and room-temperature CL spectra were measured on several GaAs nanowires and Si-doped GaAs planar layers with various doping levels, see Figures 2 and 3.

The spectra present a single peak across the band gap of GaAs $(1.424 \mathrm{eV}$ at room temperature and $1.519 \mathrm{eV}$ at very low temperature, $1.515 \mathrm{eV}$ corresponds to exciton recombination for low doping samples). We observe a blue shift of the peak energy with increasing electron concentrations (Burstein-Moss shift). It originates from the electron filling in the conduction band. As the electron Fermi level rises above the conduction band minimum, the optical absorption as well as spontaneous emission spectrum shifts to higher energies compared to the nominal band gap values. For many III-V semiconductors, the conduction band has much smaller effective density of states than the valence band. Hence the Burstein-Moss shift is typically observed for n-type doping. At lower energies, the luminescence tails also grow with increased carrier concentration as a result of Coulombic potential from ionized donor and electron-electron interaction [10]. Both effects contribute to the broadening of the CL peak with increased doping.

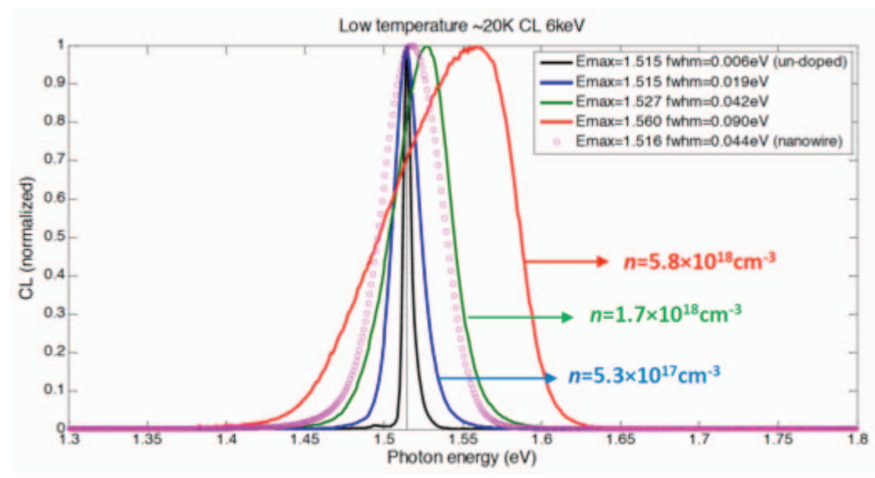

Fig. 2. Low-temperature (20K) cathodoluminescence spectra of planar GaAs layers with various n-type doping concentrations (undoped and Si-doped), and Si-doped GaAs nanowire.

Luminescence broadening could also be due to inhomogeneity and carrier heating. The homogeneity is ensured by high resolution CL mapping, as shown in Figure 1 . The effect of carrier heating was controlled by photoluminescence (PL) measurements performed on planar layers and single nanowires at various excitation intensities (not shown). PL spectra are in very good agreement with CL spectra, except in the long wavelength range were slight carrier heating is visible in CL spectra. Its contribution is negligible with respect to the total peak broadening. 


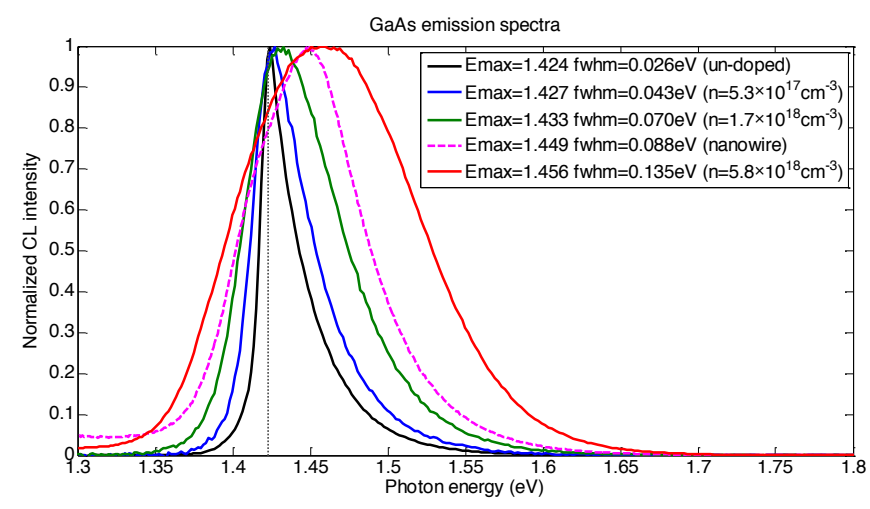

Fig. 3. Room-temperature cathodoluminescence spectra of planar GaAs layers with various n-type doping concentrations (un-doped and Si-doped), and Si-doped GaAs nanowire.

\section{Relation between CL spectra characteristics and electron concentration}

The two main characteristics of luminescence spectra are the energy position of the maximum peak (peak energy) and the full width at half maximum (FWHM). Figure 4 presents the relation between the peak energy and electron concentration from various experimental data, and Figure 5 shows the relation between FWHM and electron concentration (room temperature).

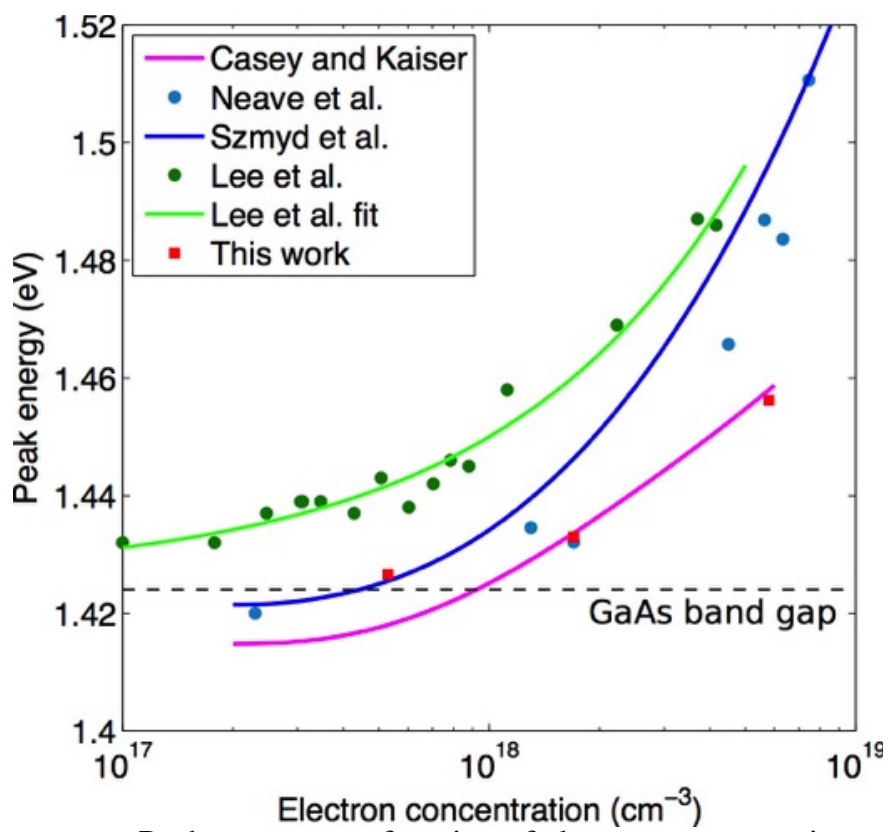

Fig. 4. Peak energy as a function of electron concentration at room temperature. Our CL measurements are compared with experimental data available in the literature: Casey and Kaiser for CL measurements on melt-grown Te-doped GaAs [11], Neave et al. for PL Si-doped GaAs grown by MBE [12], Szmyd et al. for PL Se-doped GaAs grown by MOCVD [13] and Lee et al. for PL Si-doped GaAs grown by MBE [14]. The horizontal dashed line indicates the band-gap of un-doped $\mathrm{GaAs}$ at room temperature.

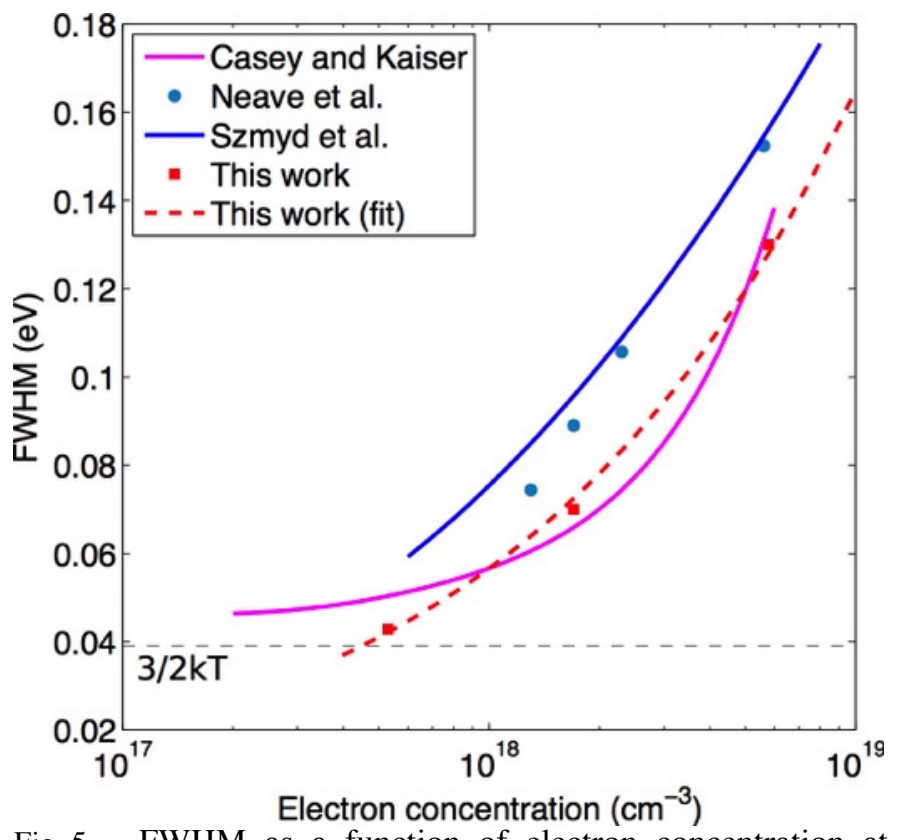

Fig. 5. FWHM as a function of electron concentration at room temperature. Our CL measurements are compared with experimental data available in the literature: Casey and Kaiser [11], Neave et al. [12] and Szmyd et al. [13]. The horizontal line indicates the room temperature thermal energy.

Our measurements are very close to that of Casey and Kaiser [11], while other data show significantly larger blue shift and broadening. Presence of acceptor states related to the growth technique may contribute to dominant radiative recombination, leading to different luminescence features. Casey and Kaiser showed very little dispersion of CL FWHM with electron concentration, so the use of FWHM to determine electron concentration seems simple and accurate [11]. The peak energy showed larger variation, especially at high doping level when the spectra tend to flatten. Therefore, we mainly focus on the use of FWHM for the determination of the n-type doping level, and the peak energy is used as a control.

At low temperature, Neave et al. observed transitions due to acceptor states for MBE-grown Si-doped GaAs samples [12]. In our Si-doped samples, we see no evidence of emission in the lower energy part of the CL spectra (Figure 2). De-Sheng et al. investigated the impact of the low-temperature FWHM with electron concentration $n$ and found a dependence in $n^{2 / 3}$ [15]. We fit the low-temperature FWHM as a power function of $n$ (Figure 6) and find the power factor very close to 2/3, which can be explained by the electron Fermi level above the parabolic portion of the conduction band [15]. 


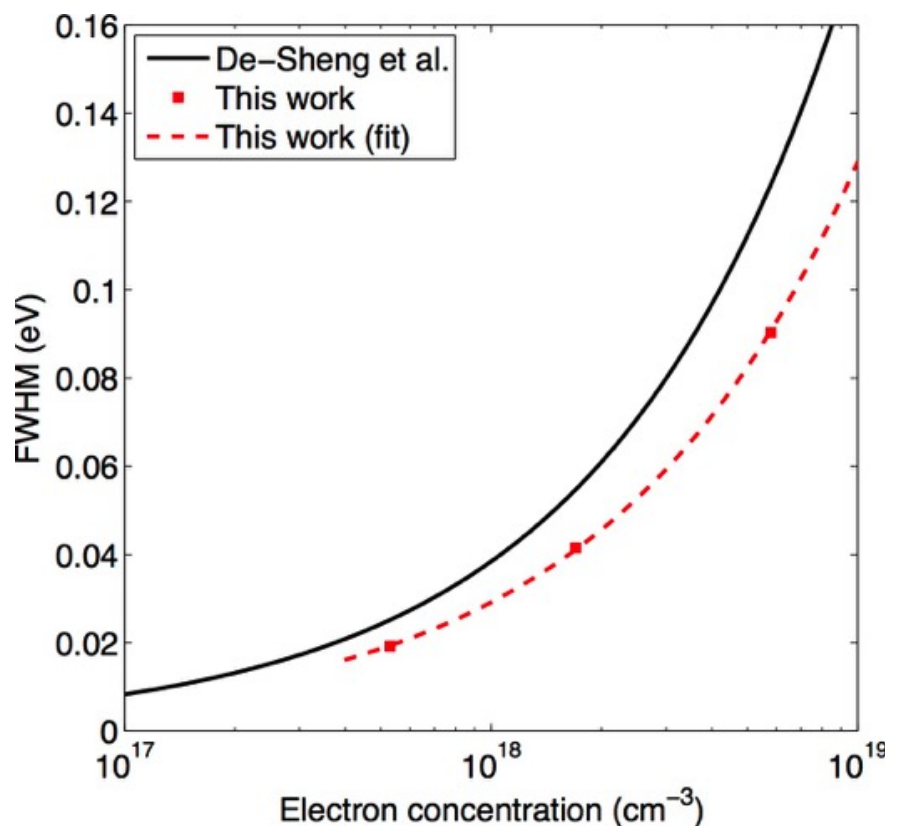

Fig. 6. FWHM as a function of electron concentration at low temperature $(20 \mathrm{~K})$. Our CL measurements are compared with experimental data from De-Sheng et al. for PL MBE GaAs:Te at $1.8 \mathrm{~K}[15]$.

\section{Determination of n-type doping in single GaAs nanowires}

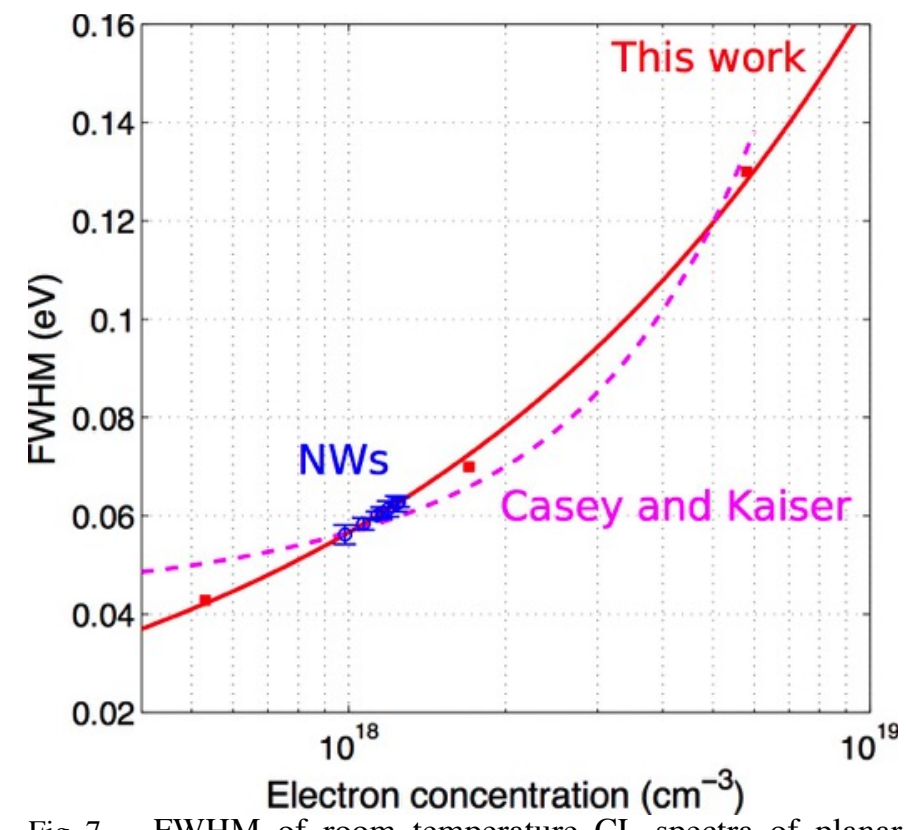

Fig. 7. FWHM of room temperature CL spectra of planar GaAs layers as a function of their electron concentration determined by Hall measurements (red marks). FWHM of CL spectra measured on single GaAs nanowires are compared to planar layers in order to assess the n-type doping level $\sim 1$ $2 \times 10^{18} \mathrm{~cm}^{-3}$ (blue circles and error bars).
The measured FWHM can now be used to determine the electron concentration of nanowires. We checked that there is no clear unexpected luminescence peak and the position of the maximal intensity is blue shifted. Figure 7 reports the roomtemperature FWHM of CL spectra measured on planar Sidoped GaAs layers (red). They are used as a reference to determine the electron concentration of different nanowires of the same sample based on the FWHM of the emission peak (blue marks). Doping levels of Si-doped nanowires are of the order of $1 \times 10^{18} \mathrm{~cm}^{-3}$ to $2 \times 10^{18} \mathrm{~cm}^{-3}$. Using the spectral shape of luminescence, we then provide a robust way to assess the ntype doping level above degeneration threshold $\left(\sim 5 \times 10^{17} \mathrm{~cm}^{-3}\right)$. More accurate estimation can be achieved by fitting the whole spectrum with physical model $[16,17]$.

\section{CONCLUSION}

In this work, high-resolution cathodoluminescence mapping of single GaAs nanowires has been performed at low (20K) and room temperature. Cathodoluminescence spectra were compared to planar Si-doped GaAs layers whose doping levels are determined by Hall measurements. The spectral shift and the broadening of luminescence spectra are a signature of an increased density of electrons. They are compared to experimental data available in the literature. We infer n-type doping of $1 \times 10^{18} \mathrm{~cm}^{-3}$ to $2 \times 10^{18} \mathrm{~cm}^{-3}$, with a high spatial homogeneity along the nanowire.

This method could be extended p-type nanowires, and polycrystalline thin films developed for photovoltaic applications. It provides an alternative way to probe carrier concentration in nanostructured and polycrystalline semiconductors, and to map the spatial inhomogeneity of dopants.

\section{ACKNOWLEDGMENTS}

This work was partly supported by the French ANR projects NANOCELL (ANR-RF-2015-01) and HETONAN. The Attolight cathodoluminescence tool was funded by public grants supported by the Region Ile-de-France in the framework of C'Nano IdF (nanoscience competence center of Paris Region), by the European Union (FEDER 2007-2013), and by the Labex GANEX (ANR-11-LABX-0014) and NanoSaclay (ANR-10-LABX-0035) as part of the "Investissements d'Avenir" program managed by the French National Research Agency (ANR).

\section{REFERENCES}

[1] Gaute Otnes, Magnus T. Borgström, "Towards high efficiency nanowire solar cells," Nano Today, in press (DOI: 10.1016/j.nantod.2016.10.007), 2017. 
[2] M. Yao et al., "Tandem Solar Cells Using GaAs Nanowires on Si: Design, Fabrication, and Observation of Voltage Addition," NanoLetters, vol. 15, no. 11, pp. 7217-7224, 2015.

[3] R. R. LaPierre, "Numerical model of current-voltage characteristics and efficiency of GaAs nanowire solar cells," Journal of Applied Physics, vol. 109, no. 3, p. 034311, Feb. 2011.

[4] M. Yao et al., "GaAs Nanowire Array Solar Cells with Axial pi-n Junctions," Nano Lett., vol. 14, no. 6, pp. 3293-3303, juin 2014.

[5] K. Storm et al., "Spatially resolved Hall effect measurement in a single semiconductor nanowire," Nat Nano, vol. 7, no. 11, pp. 718-722, Nov. 2012

[6] O. Hultin, G. Otnes, M. T. Borgström, M. Björk, L. Samuelson, and K. Storm, "Comparing Hall Effect and Field Effect Measurements on the Same Single Nanowire," Nano Lett., vol. 16, no. 1, pp. 205-211, Jan. 2016.

[7] S. Arab, M. Yao, C. Zhou, P. D. Dapkus, and S. B. Cronin, "Doping concentration dependence of the photoluminescence spectra of n-type GaAs nanowires," Applied Physics Letters, vol. 108, no. 18, p. 182106, May 2016.

[8] H. J. Joyce, J. L. Boland, C. L. Davies, S. A. Baig, and M. B. Johnston, "A review of the electrical properties of semiconductor nanowires: insights gained from terahertz conductivity spectroscopy," Semicond. Sci. Technol., vol. 31, no. 10, p. 103003, 2016.

[9] F. Glas, J.-C. Harmand, and G. Patriarche, "Why Does Wurtzite Form in Nanowires of III-V Zinc Blende Semiconductors?," Phys. Rev. Lett., vol. 99, no. 14, p. 146101, Oct. 2007.
[10] E. O. Kane, "Band tails in semiconductors," Solid-State Electronics, vol. 28, no. 1, pp. 3-10, Jan. 1985.

[11] H. C. Casey and R. H. Kaiser, "Analysis of N Type GaAs with Electron Beam Excited Radiative Recombination," $J$. Electrochem. Soc., vol. 114, no. 2, pp. 149-153, Feb. 1967.

[12] J. H. Neave, P. J. Dobson, J. J. Harris, P. Dawson, and B. A. Joyce, "Silicon doping of MBE-grown GaAs films," Appl. Phys. A, vol. 32, no. 4, pp. 195-200, Dec. 1983.

[13] M. Szmyd, P. Porro, A. Majerfeld, and S. Lagomarsino, "Heavily doped GaAs:Se. I. Photoluminescence determination of the electron effective mass," Journal of Applied Physics, vol. 68, no. 5, pp. 2367-2375, Sep. 1990.

[14] N.-Y. Lee et al., "Determination of conduction band tail and Fermi energy of heavily Si-doped GaAs by room-temperature photoluminescence," Journal of Applied Physics, vol. 78, no. 5, pp. 3367-3370, Sep. 1995.

[15] De-Sheng, Y. Makita, K. Ploog, and H. J. Queisser, "Electrical properties and photoluminescence of Te-doped GaAs grown by molecular beam epitaxy," Journal of Applied Physics, vol. 53, no. 2, pp. 999-1006, Feb. 1982.

[16] Wurfel, "The chemical potential of radiation," J. Phys. C: Solid State Phys., vol. 15, no. 18, p. 3967, Jun. 1982.

[17] J. K. Katahara and H. W. Hillhouse, "Quasi-Fermi level splitting and sub-bandgap absorptivity from semiconductor photoluminescence," J. Appl. Phys., vol. 116, no. 17, p. 173504, 2014. 